\title{
Renormalization Constant of the Color Gauge Field as a Probe of Confinement
}

\author{
Masud Chaichian \\ Department of Physics, High Energy Physics Division \\ University of Helsinki \\ and \\ Helsinki Institute of Physics \\ P.O. Box 9, FIN-00014 Helsinki, Finland \\ and \\ Kazuhiko Nishijima \\ Nishina Memorial Foundation \\ 2-28-45 Honkomagome, Bunkyo-ku, Tokyo 113-8941, Japan
}

\begin{abstract}
The mechanism of color confinement as a consequence of an unbroken non-abelian gauge symmetry and asymptotic freedom is elucidated and compared with that of other models based on an analogy with the type II superconductor. It is demonstrated that a sufficient condition for color confinement is given by $Z_{3}^{-1}=0$ where $Z_{3}$ denotes the renormalization constant of the color gauge field. It is shown that this condition is actually satisfied in quantum chromodynamics and that some of the characteristic features of other models follow from it.
\end{abstract}

\section{Introduction}

It is our consensus that strong interactions are governed by quantum chromodynamics (QCD) or the gauge theory of quarks and gluons. These fundamental constituents of hadrons carry the color quantum number and are considered to be unobservable. This 
is a conclusion drawn from our unsuccessful attempts to observe isolated quarks and is referred to as color confinement - abbreviated as c.c. - in what follows. It is the subject of this paper to compare various interpretations of c.c. in an effort to extract common features from them. For this purpose we review early attempts to interpret confinement in Section 2. These theories are formulated in configuration space and are characterized by the two phase structure or the dual Meissner effect with a finite penetration depth. In Section 3 we recapitulate an interpretation of confinement formulated in the state vector space on the basis of BRS invariance and asymptotic freedom (AF) skipping details of the proof. In this section we present two alternative conditions for confinement. In Section 4 we prove that one of these two conditions is a consequence of the other on the basis of the renormalization group ( $R G)$ method. It is shown that c.c. is realized when the condition $Z_{3}^{-1}=0$ is satisfied where $Z_{3}$ is the renormalization constant of the color gauge field. It is called the condition for color confinement abbreviated as CCC hereafter. In Section 5 it is proved that the CCC is really satisfied in QCD.

Finally in Section 6 we compare the consequences of the CCC with other interpretations. First, we give an intuitive interpretation of the $\mathrm{CCC}$ in fictitious electrodynamics. Next, we discuss the connection between the CCC and the linear potential between a heavy quark and a heavy antiquark pair resulting from Wilson's area law. Then, we show that the flux of the color gauge field emerging from color singlet hadrons cannot penetrate into the confining vacuum leaving no trace of long range forces. This resembles the dual Meissner effect introduced in some of the other interpretations.

Two appendices are included. Appendix A is intended to clarify the relationship between two alternative forms of the conditions for color confinement. Appendix B gives a derivation of (3.48) in the absence of asymptotic fields due to infrared singularities.

\section{Early Attempts to Interpret Confinement}

Because of its profound mysterious nature exhibited in strong interactions various attempts have been made to understand the mechanism of color confinement on the basis of classical or semi-classical gauge theories eventually exploiting topological quantization.

Starting from a classical Lagrangian of the Higgs model, Nielsen and Olesen [1] identified the hadronic strings with the Landau-Ginzburg-Abrikosov vortices of quantized magnetic flux in the superconducting vacuum. Their vortices are either endless or closed and the energy of the system is minimized for a certain optimum radius of the vortex, and the total flux is topologically quantized.

Nambu [2] introduced Dirac's monopoles into this theory and realized finite vortices by putting monopoles at both ends, and Dirac's quantization for monopoles matches the flux quantization. Thus a hadronic string is formed in a superconducting vacuum by joining a monopole-antimonopole pair by a vortex of a quantized magnetic flux. The flux 
cannot spread out and the energy of the flux is proportional to the length of the string. This pair may be regarded as a meson and monopoles in the superconducting vacuum are then confined. An ordinary superconductor is a coherent superposition of charged objects, but a vacuum which confines electric, instead of magnetic, monopoles may be a coherent superposition of magnetic monopoles as suggested by Mandelstam [3] and by 't Hooft [4]. Magnetic monopoles do not appear in continuum quantum electrodynamics (QED) unless they are put by hand, so that electric charges cannot be confined in QED if this interpretation should be taken for granted. They can appear in non-abelian gauge theories, however, as shown by 't Hooft [5], and therefore the Yang-Mills vacuum could be a coherent superposition of magnetic monopoles, and confinement must be a property characteristic of non-abelian gauge theories. We shall come back to this subject later again in Subsections 3.4 and 6.1.

In the quantized version of non-abelian gauge theories, the only known way of including higher order corrections in a gauge-invariant manner is to exploit the lattice gauge theory [6], although Lorentz invariance is recovered only in the limit of the vanishing lattice constant. Since confinement is a non-perturbative effect it is important to check it in the lattice gauge theory. Wilson [6] has formulated confinement in the form of the area law for the loop correlation function leading to a confining linear potential between a quark and an antiquark. In the strong coupling approximation the area law is obeyed even in QED, but it is a non-trivial problem to check if the strong coupling regime could be continued to the weak coupling one without encountering a phase transition. Susskind and Kogut [7] analyzed the lattice gauge theory in the strong coupling approximation and found that the confining strong coupling phase resembles that of a type II superconductor with electric and magnetic fields interchanged. We shall come back to the discussion of the area law later again in the Subsection 6.2.

All such attempts including the recent supersymmetric Seiberg-Witten theory [8] tend to indicate that confinement is a consequence of the coherent superposition of magnetic monopoles in the vacuum state dual to the superconducting one based on coherent superposition of charged objects such as Cooper pairs. Quarks and antiquarks are joined together by the electric flux penetrating into the vacuum or through the normal conducting phase enclosed by the superconducting phase. In other words, confinement is characterized by the two-phase structure or the Meissner effect of a finite penetration depth.

In electrodynamics all the physical media are characterized by their dielectric constant $\epsilon>1$. Suppose that there is a fictitious medium of dielectric constant [9],

$$
\epsilon \ll 1 \text {. }
$$

This medium is antiscreening. When a small charge is placed in this medium, it will crack and develop a hole surrounding this charge. We have $\epsilon=1$ inside and $\epsilon<<1$ outside. Because of the antiscreening nature of the medium the induced charge on the inner surface of the hole is of the same sign as the originally inserted charge. In order to reduce the 
size of the hole the repulsion between the original charge and the induced one must be overcome, but elimination of the hole would require infinite energy, so that the hole will not disappear. The two media, one with $\epsilon=1$ and the other with $\epsilon<<1$, are considered to correspond to normal conducting and superconducting states, respectively, and such a two-phase structure of the vacuum is common to all the models constructed in analogy with the type II superconductor in the configuration space.

In the next section we shall recapitulate the arguments based on the BRS invariance $[10]$ and asymptotic freedom $[11,12]$ in the state vector space.

\section{Color Confinement as a Renormalization Effect}

In a covariant quantization of gauge fields introduction of indefinite metric is indispensable. Thus the resulting state vector space $\mathcal{V}$ involves unphysical states of indefinite metric and we have to find a criterion to select physical states out of $\mathcal{V}$. For this purpose we employ the Lorentz condition in QED as a subsidiary condition, but it is more involved in nonabelian gauge theories. In what follows we shall confine ourselves to QCD, and in order to fix the notation we start from its Lagrangian density in the Pauli metric,

$$
\mathcal{L}=\mathcal{L}_{\text {inv }}+\mathcal{L}_{g f}+\mathcal{L}_{F P}
$$

where

$$
\begin{aligned}
& \mathcal{L}_{i n v}=-\frac{1}{4} F_{\mu \nu} \cdot F_{\mu \nu}-\bar{\psi}\left(\gamma_{\mu} D_{\mu}+m\right) \psi, \\
& \mathcal{L}_{g f}=A_{\mu} \cdot \partial_{\mu} B+\frac{\alpha}{2} B \cdot B \\
& \mathcal{L}_{F P}=i \partial_{\mu} \bar{c} \cdot D_{\mu} c .
\end{aligned}
$$

We have suppressed the color and flavor indices above. The first term in (3.1) is the gauge-invariant term, the second one the gauge-fixing term and the last one the FaddeevPopov ghost term. In (3.2b) $\alpha$ denotes the gauge parameter and $B$ the Nakanishi-Lautrup auxiliary field, and in (3.2c) the hermitian scalar fields $c$ and $\bar{c}$ are anticommuting and are called Faddeev-Popov (FP) ghost fields.

In what follows we shall introduce the inner and outer products of two colored objects:

$$
\begin{aligned}
& S \cdot T=\sum_{a} S^{a} T^{a}, \\
& (S \times T)^{a}=\sum_{b, c} f_{a b c} S^{b} T^{c},
\end{aligned}
$$


where $a, b, c$ etc. are color indices and $f_{a b c}$ the structure constant of the algebra $\mathrm{su}(3)$ corresponding to the color gauge group. Then covariant derivatives are defined by

$$
\begin{aligned}
& D_{\mu} \psi=\left(\partial_{\mu}-i g T \cdot A_{\mu}\right) \psi, \\
& D_{\mu} c^{a}=\partial_{\mu} c^{a}+g\left(A_{\mu} \times c\right)^{a}, \\
& F_{\mu \nu}^{a}=\partial_{\mu} A_{\nu}^{a}-\partial_{\nu} A_{\mu}^{a}+g\left(A_{\mu} \times A_{\nu}\right)^{a} .
\end{aligned}
$$

The local gauge invariance is respected by (3.2a) but not by the other two, (3.2b) and (3.2c), introduced for quantization. It so happens, however, that the total Lagrangian density is invariant under new global transformations called the Becchi-Rouet-Stora (BRS) transformations [10].

\subsection{BRS transformations}

Let us consider an infinitesimal gauge transformation of the gauge and quark fields and replace the infinitesimal gauge function either by $c$ or $\bar{c}$. They define two kinds of BRS transformations denoted by $\delta$ and $\bar{\delta}$, respectively.

$$
\begin{gathered}
\delta A_{\mu}=D_{\mu} c, \quad \bar{\delta} A_{\mu}=D_{\mu} \bar{c} \\
\delta \psi=i g(c \cdot T) \psi, \quad \bar{\delta} \psi=i g(\bar{c} \cdot T) \psi .
\end{gathered}
$$

For the auxiliary fields $B, c$ and $\bar{c}$ local gauge transformations are not even defined, but their BRS transformations can be introduced by requiring the invariance of the local Lagrangian density, namely,

$$
\delta \mathcal{L}=\bar{\delta} \mathcal{L}=0
$$

then we find the following transformations:

$$
\begin{aligned}
& \delta B=0, \delta \bar{c}=i B, \delta c=-\frac{1}{2} g(c \times c), \\
& \bar{\delta} \bar{B}=0, \bar{\delta} c=i \bar{B}, \bar{\delta} \bar{c}=-\frac{1}{2} g(\bar{c} \times \bar{c}),
\end{aligned}
$$

where $\bar{B}$ is defined by

$$
B+\bar{B}-i g(c \times \bar{c})=0 .
$$

Noether's theorem states that the BRS invariance of the Lagrangian density leads to conservation laws and we introduce two conserved BRS charges $Q_{B}$ and $\bar{Q}_{B}$ by

$$
\delta \phi=i\left[Q_{B}, \phi\right]_{\mp}, \quad \bar{\delta} \phi=i\left[\bar{Q}_{B}, \phi\right]_{\mp},
$$


where we choose the $-(+)$ sign, when the field $\phi$ is of an even (odd) power in the ghost fields $c$ and $\bar{c}$.

Maxwell's equations for the gauge field can be expressed in terms of BRS transformations as

$$
\partial_{\mu} F_{\mu \nu}+g J_{\nu}=i \delta \bar{\delta} A_{\nu}
$$

where $J_{\nu}$ denotes the color current density and $g$ the gauge coupling constant. This set of equations has been derived from the Lagrangian density (3.1), but the form of these equations is valid for a wider class of theories such as supersymmetric and grand unified theories as long as the original gauge symmetry is respected. The only deviation from the original theory represented by (3.1) appears when we try to express the color current density $J_{\nu}$ explicitly in terms of the elementary component fields.

The r.h.s. of Eq. (3.14) represents a conserved current and stands for the deviation from Maxwell's classical equations.

$$
\partial_{\mu}\left(i \delta \bar{\delta} A_{\mu}\right)=0
$$

The BRS charges are hermitian and nilpotent, for example,

$$
Q_{B}^{\dagger}=Q_{B}, \quad Q_{B}^{2}=0 .
$$

The nilpotency implies introduction of indefinite metric and a physical state $|f\rangle$ is defined by a constraint [14]

$$
Q_{B}|f\rangle=0, \quad|f\rangle \in \mathcal{V} .
$$

The collection of physical states including the vacuum state $|0\rangle$ forms the physical subspace of $\mathcal{V}$ denoted by $\mathcal{V}_{\text {phys }}$,

$$
\mathcal{V}_{\text {phys }}=\left\{|f\rangle: Q_{B}|f\rangle=0,|f\rangle \in \mathcal{V}\right\} .
$$

It should be mentioned that the $\mathrm{S}$ matrix is BRS invariant,

$$
\delta S=i\left[Q_{B}, S\right]=0,
$$

so that the physical subspace $\mathcal{V}_{\text {phys }}$ is an invariant subspace of the $\mathrm{S}$ matrix.

Furthermore, we introduce a subspace of $\mathcal{V}$ called the daughter subspace $\mathcal{V}_{d}$ defined by

$$
\mathcal{V}_{d}=\left\{|f\rangle:|f\rangle=Q_{B}|g\rangle,|g\rangle \in \mathcal{V}\right\} .
$$

Because of the nilpotency of $Q_{B}, \mathcal{V}_{d}$ is a subspace of $\mathcal{V}_{\text {phys }}$,

$$
\mathcal{V}_{d} \subset \mathcal{V}_{\text {phys }}
$$


and we introduce the Hilbert space $\mathcal{H}$ by

$$
\mathcal{H}=\mathcal{V}_{\text {phys }} / \mathcal{V}_{d}
$$

which may be called the BRS cohomology $[15,16,17]$.

\subsection{Relation to QED}

QED is the oldest example of gauge theories so that the above formulation should be applicable to it. We recognize that the subsidiary condition (3.17) to select physical states looks completely different from the Lorentz condition,

$$
B^{(+)}(x)|f\rangle=0
$$

so that we shall clarify the relationship between them.

In QED or in an abelian gauge theory the auxiliary fields $B, c$ and $\bar{c}$ are free and massless, namely,

$$
\square B(x)=\square c(x)=\square \bar{c}(x)=0 .
$$

Furthermore, in the conventional treatment of QED the ghost fields do not participate in the game so that we introduce constrained physical states [18] in terms of the positive frequency parts of ghost fields by

$$
\begin{gathered}
Q_{B}\left|f^{\prime}\right\rangle=0, \\
c^{(+)}(x)\left|f^{\prime}\right\rangle=\bar{c}^{(+)}(x)\left|f^{\prime}\right\rangle=0,
\end{gathered}
$$

and the corresponding subspace $\mathcal{V}_{\text {phys }}^{\prime}$. Consistency among them requires the following condition:

$$
\left\{Q_{B}, \bar{c}^{(+)}(x)\right\}\left|f^{\prime}\right\rangle=B^{(+)}(x)\left|f^{\prime}\right\rangle=0,
$$

which is precisely the Lorentz condition.

On the other hand, when we have the Lorentz condition and (3.25) the condition (3.17) follows automatically from the structure of $Q_{B}$ in QED, namely,

$$
Q_{B}=\int d^{3} x\left[B^{(-)}(x) \dot{c}^{(+)}(x)-\dot{B}^{(-)}(x) c^{(+)}(x)+\dot{c}^{(-)}(x) B^{(+)}(x)-c^{(-)}(x) \dot{B}^{(+)}(x)\right] .
$$

Thus we realize that the two conditions (3.17) and (3.23) are equivalent under the constraints (3.25). When we define the constrained daughter space $\mathcal{V}_{d}^{\prime}$ as a subspace of $\mathcal{V}_{d}$ constrained by (3.25), we have 


$$
\mathcal{V}_{\text {phys }}^{\prime} / \mathcal{V}_{d}^{\prime}=\mathcal{V}_{\text {phys }} / \mathcal{V}_{d}=\mathcal{H}
$$

This argument indicates that the condition (3.17) is not alien to QED [19].

\subsection{Interpretation of color confinement}

When single-quark states and single-gluon states are not physical, they are unobservable and hence confined. Thus the problem of color confinement is settled if we could prove

$$
\left.Q_{B}|q u a r k\rangle \neq 0, \quad Q_{B} \mid \text { gluon }\right\rangle \neq 0 .
$$

This is a definition of c.c. given in terms of unobservable quantities so that we shall present an alternative definition of c.c. in terms of observable quantities in Section 5.

In order to study the condition on which the relations (3.29) are satisfied we start from an identity:

$$
\begin{aligned}
\left\langle A_{\mu}^{a}(x), B^{b}(y)\right\rangle & \equiv\left\langle 0\left|T\left[A_{\mu}^{a}(x), B^{b}(y)\right]\right| 0\right\rangle \\
& =-\delta_{a b} \partial_{\mu} D_{F}(x-y),
\end{aligned}
$$

where $a$ and $b$ denote color indices and $D_{F}$ is the free massless propagator. This identity implies that both $A_{\mu}$ and $B$ generate a massless spin zero particle as applied to the vacuum state. Thus their asymptotic fields may be expressed as

$$
A_{\mu}^{a}(x)^{i n}=\alpha_{\mu}^{a}(x)+\partial_{\mu} \chi^{a}(x), \quad B^{b}(y)^{i n}=\beta^{b}(y),
$$

where $\alpha_{\mu}$ denotes the incoming gluon field and $\chi$ and $\beta$ are the incoming fields of the massless spin zero particle. Here, we have assumed the validity of the Lehmann-SymanzikZimmermann (LSZ) asymptotic condition [20] or its suitable modification so that we can relate a field operator to a particle state through its asymptotic field. Since QCD is infested with infrared singularities an infrared cut-off is introduced to validate the asymptotic condition, and only after confinement of colored particles it can be lifted safely for the system of hadrons. This is related to the Meissner-like effect discussed in Subsection 6.3.

The asymptotic fields introduced above satisfy the relations,

$$
\begin{gathered}
\partial_{\mu} \alpha_{\mu}^{a}=0, \quad \square^{2} \alpha_{\mu}^{a}=0, \\
\square \chi^{a}=\alpha \cdot \beta^{a}, \quad \square \beta^{a}=0,
\end{gathered}
$$

and

$$
\begin{gathered}
\left\langle\chi^{a}(x), \beta^{b}(y)\right\rangle=-\delta_{a b} D_{F}(x-y), \\
\left\langle\beta^{a}(x), \beta^{b}(y)\right\rangle=0,
\end{gathered}
$$


Now we shall study the implications of c.c. in the properties of the asymptotic fields. Assume that $\alpha_{\mu}^{a}$ is BRS invariant, namely,

$$
\delta \alpha_{\mu}^{a}=0
$$

then

$$
\left.Q_{B} \mid \text { gluon }\right\rangle=Q_{B} \alpha_{\mu}^{a}|0\rangle=(-i) \delta \alpha_{\mu}^{a}|0\rangle=0,
$$

so that a single-gluon state is physical and hence observable. Therefore, c.c. implies

$$
\delta \alpha_{\mu}^{a} \neq 0 .
$$

Now

$$
\begin{aligned}
\delta A_{\mu}^{a, i n} & =\delta \alpha_{\mu}^{a}+\partial_{\mu}\left(\delta \chi^{a}\right) \\
& =\partial_{\mu} c^{a, i n}+g\left(A_{\mu} \times c\right)^{a, i n} .
\end{aligned}
$$

When the second bilinear term on the r.h.s. of Eq. (3.38) is absent, there is no asymptotic field of unit spin in this expression and we are led to Eq. (3.36). This is really the case in perturbation theory. Therefore, a necessary condition for gluon confinement is the nonvanishing of $\left(A_{\mu} \times c\right)^{i n}$. This means that there must be a bound state between a gluon and a FP ghost, and then and only then we have (3.37) and gluons are confined. Similarly, quarks are confined when a quark and a FP ghost form a bound state. Thus the problem of confinement reduces to that of bound states.

One of the present authors (KN) and Okada studied the bound state problem by making use of the Bethe-Salpeter equations in the ladder approximation and recognized that quarks and probably also gluons are confined when two FP ghosts form a bound state [21]. The statement that c.c. is a consequence of the formation of the dighost bound state was plausible but not conclusive because of the approximate nature of the above treatment. This condition was further refined and reappeared later in a simpler form, namely, the condition (3.49) to be introduced in the next subsection. It is not difficult to show that the formation of dighost bound states is a consequence of this new condition. This relationship is clarified in Appendix A.

\subsection{Conditions for color confinement}

By utilizing the conserved current (3.15) we shall introduce a set of Ward-Takahashi identities. First, we define the conserved color charge $Q^{a}$ in terms of the color current density $J_{\nu}^{a}$ in Eq. (3.14) by

$$
Q^{a}=\int d^{3} x J_{0}^{a}(x) .
$$

Then let us consider a colored field $\Phi^{\alpha}$ belonging to an irreducible representation $R^{a}$ of the Lie algebra $\mathrm{su}(3)$ of the color symmetry,

$$
\begin{aligned}
& {\left[\Phi^{\alpha}(y), Q^{a}\right]=R_{\alpha \beta}^{a} \Phi^{\beta}(y),} \\
& {\left[\bar{\Phi}^{\alpha}(z), Q^{a}\right]=-\bar{\Phi}^{\beta}(z) R_{\beta \alpha}^{a}=-\left(R^{a}\right)_{\alpha \beta}^{T} \bar{\Phi}^{\beta}(z),}
\end{aligned}
$$


where $\bar{\Phi}$ is the adjoint of $\Phi$ and $R^{a}$ is an hermitian matrix.

For the quark field $R^{a}$ is given by $T^{a}$ in Eq. (3.5) or $\lambda^{a} / 2$, where $\lambda^{a}$ denotes GellMann's matrix. For the color gauge field obeying the adjoint representation we have $R_{b c}^{a}=-i f_{a b c}$. Then we have a set of Ward-Takahashi identities of the form

$$
\begin{aligned}
& \partial_{\mu}\left\langle\delta \bar{\delta} A_{\mu}^{a}(x), \Phi^{\alpha}(y), \bar{\Phi}^{\beta}(z)\right\rangle \\
& =i g\left[R_{\alpha \gamma}^{a} \delta^{4}(x-y)\left\langle\Phi^{\gamma}(y), \bar{\Phi}^{\beta}(z)\right\rangle-R_{\delta \beta}^{a} \delta^{4}(x-z)\left\langle\Phi^{\alpha}(y), \bar{\Phi}^{\delta}(z)\right\rangle\right] .
\end{aligned}
$$

This identity follows from Maxwell's equations. When $\Phi$ represents a color singlet field we have $R=0$ and the r.h.s. of Eq. (3.41) vanishes identically.

In order to derive a condition for color confinement we need some preparations. When an operator $M$ is the BRS transform of another operator $N$, namely,

$$
M=\delta N=i\left[Q_{B}, N\right]_{\mp},
$$

$M$ is called an exact operator. Thus its matrix element between two physical states $|\alpha\rangle$ and $|\beta\rangle$ vanishes identically because of the definition of the physical states (3.17).

$$
\langle\beta|M| \alpha\rangle=0 .
$$

Therefore, when the expectation value of an exact operator $M$ in a state $|\gamma\rangle$ does not vanish, namely,

$$
\langle\gamma|M| \gamma\rangle \neq 0
$$

it is an indication that the state $|\gamma\rangle$ is unphysical,

$$
Q_{B}|\gamma\rangle \neq 0
$$

We are going to exploit this fact in order to derive (3.29). For this purpose we shall remove $\partial_{\mu}$ in Eq. (3.41). In momentum space $\partial_{\mu}$ denotes the momentum transfer so that we differentiate the Fourier transform of Eq. (3.41) with respect to the momentum transfer and take the limit of zero momentum transfer. We shall illustrate this procedure in QED by starting from the following Ward-Takahashi identity:

$$
(p-q)_{\mu} \Gamma_{\mu}(p, q)=-i\left(S_{F}^{-1}(p)-S_{F}^{-1}(q)\right) .
$$

We differentiate this identity with respect to $p_{\mu}$ and then take the limit of $q \rightarrow p$ and obtain

$$
\Gamma_{\mu}(p, p)=-i \frac{\partial}{\partial p_{\mu}} S_{F}^{-1}(p) .
$$

This derivation is valid provided that $\Gamma_{\mu}(p, q)$ does not have a pole at $(p-q)^{2}=0$. 
Now define the spin zero projection of $\delta \bar{\delta} A_{\mu}$ and denote it by $M_{\mu}$, then apply the above procedure to a colored field $\Phi^{\alpha}$, then we have

$$
\left\langle p, \beta\left|M_{\mu}^{a}\right| p, \alpha\right\rangle \propto R_{\beta \alpha}^{a} \times(\text { kinematical factor }),
$$

where $|p, \alpha\rangle$ denotes a state involving a quantum of $\Phi^{\alpha}$ with four-momentum $p$. In this derivation, however, we have assumed the absence of the massless pole as in QED, and this assumption is expressed by [22]

$$
\delta \bar{\delta} \chi^{a}=0 .
$$

The derivation of (3.48) in the absence of the asymptotic fields is discussed in Appendix B.

Then we can refer to the argument based on (3.44) and (3.45) and we conclude that the quantum of the colored field $\Phi^{\alpha}$ is confined. For a color singlet field we have $R=0$ and its quantum is not confined just as in the case of (3.36). Thus we may conclude that Eq. (3.49) is a sufficient condition for c.c. in the sense of (3.29).

At this stage we quote some examples in which this condition is not satisfied. The first example is an abelian gauge theory represented by QED. In this case the quanta of the FP ghost fields are free so that they cannot form bound states with charged particles, and charge confinement cannot be realized. We can also give an alternative explanation: In this case we can easily derive

$$
i \delta \bar{\delta} \chi=-\beta,
$$

and the condition (3.49) is not satisfied.

Another example is found when a certain gauge symmetry is spontaneously broken. In analogy with Eq. (3.46) we may express the identity (3.41) for the quark field in momentum space as

$$
(p-q)_{\mu} V_{\mu}^{a}(p, q)=-i g T_{\alpha \beta}^{a}\left[S_{F}^{-1}(p, \alpha)-S_{F}^{-1}(q, \beta)\right] .
$$

Assume that the gauge symmetry corresponding to the group index $a$ is broken and that the quark masses for the colors $\alpha$ and $\beta$ are non-degenerate. In the limit $q \rightarrow p$ the r.h.s. does not vanish because of the mass difference,

$$
S_{F}^{-1}(p, \alpha)-S_{F}^{-1}(p, \beta) \neq 0
$$

This implies that $V_{\mu}^{a}(p, q)$ should develop a massless pole. Physically the appearance of this pole is a signal that the Nambu-Goldstone boson has emerged in the form

$$
\delta \bar{\delta} \chi^{a} \neq 0, \square \delta \bar{\delta} \chi^{a}=0
$$


which violates the condition (3.49).

These two cases serve to explain why the electroweak interactions do not confine any particles. Indeed, the electroweak gauge theory is constructed on the gauge group $S U(2) \times$ $U(1)$, but this symmetry is spontaneously broken and reduced to $\mathrm{U}(1)$ corresponding to the electromagnetic gauge symmetry. Thus we are left with only an abelian gauge symmetry and this explains why the electroweak interactions do not lead us to confinement.

\subsection{Superconvergence relation}

Quite independently of (3.49) a condition for gluon confinement has been derived from the study of the renormalization constant of the color gauge field.

Let us introduce the propagator of the color gauge field,

$$
\left\langle A_{\mu}^{a}(x) A_{\nu}^{b}(y)\right\rangle=\frac{-i}{(2 \pi)^{4}} \delta_{a b} \int d^{4} k e^{i k \cdot(x-y)} D_{F}(k)_{\mu \nu}
$$

where

$$
D_{F}(k)_{\mu \nu}=\left(\delta_{\mu \nu}-\frac{k_{\mu} k_{\nu}}{k^{2}-i \epsilon}\right) D\left(k^{2}\right)+\alpha \frac{k_{\mu} k_{\nu}}{\left(k^{2}-i \epsilon\right)^{2}} .
$$

We introduce the Lehmann representation [23] for $D\left(k^{2}\right)$ by

$$
D\left(k^{2}\right)=\int d m^{2} \frac{\rho\left(m^{2}\right)}{k^{2}+m^{2}-i \epsilon},
$$

then Lehmann's theorem gives

$$
Z_{3}^{-1}=\int d m^{2} \rho\left(m^{2}\right)
$$

where $Z_{3}$ is the renormalization constant of the color gauge field. Oehme and Zimmermann [24] have studied the structure of this propagator in the Landau gauge and have proved the superconvergence relation

$$
Z_{3}^{-1}=\int d m^{2} \rho\left(m^{2}\right)=0,
$$

when the number of quark flavors $N_{f}$ is less than 10, namely, in the case 1) of Subsection 5.3 as we shall see later. This proof is based on the renormalization group (RG) method, and we shall come back to this method later. Then it was recognized by one of the present authors $(\mathrm{KN})$ and also by Oehme that gluon confinement follows from this superconvergence relation $[25,26]$.

Thus it turns out to be an important problem to clarify the relationship between these two conditions (3.49) and (3.58) and we shall study it in the next section. 


\section{Renormalization Group and Color Confinement}

In the preceding section we have obtained two kinds of conditions for c.c., and in this section we elucidate their relationship in order to find the most fundamental condition.

\subsection{Renormalization group}

The infinitesimal operator of the RG is given by the differential operator

$$
\mathcal{D}=\mu \frac{\partial}{\partial \mu}+\beta(g) \frac{\partial}{\partial g}-2 \alpha \gamma_{V}(g, \alpha) \frac{\partial}{\partial \alpha},
$$

where $\mu$ denotes the renormalization point, $\alpha$ the gauge parameter, and $\gamma_{V}$ the anomalous dimension of the color gauge field. An element of the RG may be expressed as

$$
R(\rho)=\exp (\rho \mathcal{D}),
$$

where $\rho$ is the parameter of the RG and we have the composition law of the group,

$$
R(\rho) \cdot R\left(\rho^{\prime}\right)=R\left(\rho+\rho^{\prime}\right) .
$$

Let $Q$ be a function of $g, \alpha$ and $\mu$, and we define the running $Q$ by

$$
\begin{aligned}
\bar{Q}(\rho) & =\exp (\rho \mathcal{D}) \cdot Q(g, \alpha, \mu) \\
& =Q(\bar{g}(\rho), \bar{\alpha}(\rho), \bar{\mu}(\rho)),
\end{aligned}
$$

with the initial condition

$$
\bar{Q}(0)=Q
$$

Then introduce Green's function $G\left(p_{i} ; g, \alpha, \mu\right)$ and let its anomalous dimension be $\gamma(g, \alpha)$, then we have a $R G$ equation:

$$
[\mathcal{D}+\gamma(g, \alpha)] G\left(p_{i} ; g, \alpha, \mu\right)=0 .
$$

Its running version defined by

$$
\bar{G}(\rho)=\exp (\rho \mathcal{D}) \cdot G\left(p_{i} ; g, \alpha, \mu\right) .
$$

satisfies the following equation:

$$
\frac{\partial}{\partial \rho} \bar{G}(\rho)=-\bar{\gamma}(\rho) \bar{G}(\rho)
$$

Its integral is given by

$$
G\left(p_{i} ; g, \alpha, \mu\right)=\exp \left[\int_{0}^{\rho} d \rho^{\prime} \bar{\gamma}\left(\rho^{\prime}\right)\right] \cdot G\left(p_{i} ; \bar{g}(\rho), \bar{\alpha}(\rho), \bar{\mu}(\rho)\right)
$$


The RG provides us with the relationship between renormalized and unrenormalized expressions. In order to give a finite value to an unrenormalized expression we have to introduce a cut-off $\Lambda$, then higher order corrections tend to decrease for momentumtransfer beyond the cut-off $\Lambda$. Then we may assume that the running coupling constant $\bar{g}(\rho)$ tends to the unrenormalized one or the bare one $g_{0}$ in the limit $\rho \rightarrow \infty$,

$$
\lim _{\rho \rightarrow \infty} \bar{g}(\rho)=g_{0}
$$

provided that the cut-off $\Lambda$ is kept finite. In the RG approach we usually formulate the initial conditions by keeping $\Lambda$ finite, then we can take the limit $\rho \rightarrow \infty$ in Eq. (4.9) to obtain

$$
G\left(p_{i} ; g, \alpha, \mu\right)=\exp \left[\int_{0}^{\infty} d \rho^{\prime} \bar{\gamma}\left(\rho^{\prime}\right)\right] \cdot G^{(0)}\left(p_{i} ; g_{0}, \alpha_{0}, \infty\right)
$$

where $G^{(0)}$ denotes the unrenormalized Green function and the factor

$$
Z=\exp \left[-\int_{0}^{\infty} d \rho^{\prime} \bar{\gamma}\left(\rho^{\prime}\right)\right]
$$

gives the renormalization constant of Green's function $G$. In particular the gluon propagator $D\left(k^{2}\right)$ satisfies

$$
R\left(k^{2} ; g, \alpha, \mu\right)=k^{2} D\left(k^{2} ; g, \alpha, \mu\right)=1, \text { for } k^{2}=\mu^{2},
$$

since this is precisely the definition of the renormalization point $\mu$. Replacing $G$ by $R$ in (4.9) we find

$$
R\left(k^{2} ; g, \alpha, \mu\right)=\exp \left[2 \int_{0}^{\rho} d \rho^{\prime} \bar{\gamma}_{V}\left(\rho^{\prime}\right)\right] \cdot R\left(k^{2} ; \bar{g}(\rho), \bar{\alpha}(\rho), \bar{\mu}(\rho)\right) .
$$

Then, by putting $k^{2}=\bar{\mu}^{2}(\rho)$ in (4.13) and referring to (4.12) we find

$$
R\left(\bar{\mu}^{2}(\rho) ; g, \alpha, \mu\right)=\exp \left[2 \int_{0}^{\rho} d \rho^{\prime} \bar{\gamma}_{V}\left(\rho^{\prime}\right)\right] .
$$

Now insert the Lehmann representation (3.56) into the l.h.s. of Eq. (4.14) and take the limit $\rho \rightarrow \infty$ or $\bar{\mu}^{2}(\rho)=\mu^{2} \exp (2 \rho) \rightarrow \infty$, and we find, for a finite cut-off, the relation

$$
\int d m^{2} \rho\left(m^{2}\right)=\exp \left[2 \int_{0}^{\infty} d \rho^{\prime} \bar{\gamma}_{V}\left(\rho^{\prime}\right)\right] .
$$

Combing this relation with Lehmann's theorem (3.57), we find

$$
Z_{3}^{-1}=\exp \left[2 \int_{0}^{\infty} d \rho^{\prime} \bar{\gamma}_{V}\left(\rho^{\prime}\right)\right]
$$

and it satisfies the RG equation

$$
\left(\mathcal{D}+2 \gamma_{V}\right) Z_{3}^{-1}=0
$$


In the cut-off theory we first take the limit $\rho \rightarrow \infty$ and then $\Lambda \rightarrow \infty$, but in what follows we invert the order of limiting procedures by taking the limit $\Lambda \rightarrow \infty$ first. Thus some of the initial conditions introduced in the cut-off theory are not necessarily satisfied as we shall see in what follows.

\subsection{Conditions for color confinement and their relationship}

We shall go back to the condition (3.49). Since $i \delta \bar{\delta} \chi^{a}$ is a free massless field we may assume that its most general form is given by

$$
i \delta \bar{\delta} \chi^{a}=-C \beta^{a} .
$$

In $\mathrm{QED}$ we have $C=1$ and confinement requires $C=0$. Now we have to study how to determine the coefficient $C$, and we start from the following relation based on Eq. (3.34):

$$
\left\langle i \delta \bar{\delta} \chi^{a}(x), \chi^{b}(y)\right\rangle=C \delta_{a b} D_{F}(x-y) .
$$

The field $\chi$ is a complicated asymptotic field, however, and we shall express this relation in terms of Heisenberg operators. For this purpose we shall consider the two-point function

$$
\left\langle i \delta \bar{\delta} A_{\mu}^{a}(x), A_{\nu}^{b}(y)\right\rangle
$$

then because of Eq. (3.15) the most general form of its Fourier transform can be expressed as

$$
\left(\delta_{\mu \nu}-\frac{k_{\mu} k_{\nu}}{k^{2}-i \epsilon}\right) \int d m^{2} \frac{\sigma\left(m^{2}\right)}{k^{2}+m^{2}-i \epsilon}+C \frac{k_{\mu} k_{\nu}}{k^{2}-i \epsilon} .
$$

The second term corresponds to the contributions of the spin zero massless particles and we immediately obtain

$$
\partial_{\mu}\left\langle i \delta \bar{\delta} A_{\mu}^{a}(x), A_{\nu}^{b}(y)\right\rangle=i \delta_{a b} C \partial_{\nu} \delta^{4}(x-y) .
$$

Furthermore, because of (3.15) the l.h.s. can be cast in the form of an equal-time commutator,

$$
\begin{aligned}
\delta\left(x_{0}-y_{0}\right)\left\langle 0\left|\left[i \delta \bar{\delta} A_{0}^{a}(x), A_{j}^{b}(y)\right]\right| 0\right\rangle= & i \delta_{a b} C \partial_{j} \delta^{4}(x-y), \\
& (j=1,2,3) .
\end{aligned}
$$

As has been shown in $[27,28]$ this constant $C$ satisfies an RG equation

$$
\left(\mathcal{D}-2 \gamma_{F P}\right) C=0 .
$$

In order to study the equal-time commutator (4.23) we shall go back to the unrenormalized version, then

$$
i \delta \bar{\delta} A_{\nu}^{(0)}=\partial_{\mu} A_{\mu \nu}^{(0)}+g_{0} \partial_{\mu}\left(A_{\mu}^{(0)} \times A_{\nu}^{(0)}\right)+g_{0} J_{\nu}^{(0)},
$$


where $A_{\mu \nu}=\partial_{\mu} A_{\nu}-\partial_{\nu} A_{\mu}$ and the superscript (0) is attached to unrenormalized expressions. When we insert this expression into (4.23) we find that the only surviving term is given by

$$
\begin{aligned}
& \delta\left(x_{0}-y_{0}\right)\left\langle 0\left|\left[i \delta \bar{\delta} A_{0}^{a}(x)^{(0)}, A_{j}^{b}(y)^{(0)}\right]\right| 0\right\rangle \\
= & \delta\left(x_{0}-y_{0}\right)\left\langle 0\left|\left[\partial_{k} A_{k 0}^{a}(x)^{(0)}, A_{j}^{b}(y)^{(0)}\right]\right| 0\right\rangle \\
= & i \delta_{a b} a^{(0)} \partial_{j} \delta^{4}(x-y),
\end{aligned}
$$

where $a^{(0)}$ is a parameter which depends on the normalization of the gauge field. In the unrenormalized version $a^{(0)}=1$ and in the renormalized version $a=Z_{3}^{-1}$. So we find in the unrenormalized version

$$
C^{(0)}=a^{(0)}
$$

Therefore, in the cut-off theory the boundary condition for $C$ is given by

$$
\lim _{\rho \rightarrow \infty}(\bar{C}(\rho)-\bar{a}(\rho))=0
$$

The RG equations for $\bar{C}$ and $\bar{a}$ are given, respectively, by

$$
\begin{gathered}
\frac{\partial}{\partial \rho} \bar{C}(\rho)=2 \bar{\gamma}_{F P}(\rho) \bar{C}(\rho), \\
\frac{\partial}{\partial \rho} \bar{a}(\rho)=-2 \bar{\gamma}_{V}(\rho) \bar{a}(\rho) .
\end{gathered}
$$

$\gamma_{F P}$ denotes the anomalous dimension of the FP ghost fields. The formal solution of the RG equation for $\bar{C}(\rho)$ satisfying the boundary condition (4.28) is given in terms of $\bar{a}(\rho)$ by

$$
\begin{aligned}
\bar{C}(\rho)= & \bar{a}(\rho)-2 \int_{\rho}^{\infty} d \rho^{\prime}\left(\bar{\gamma}_{V}\left(\rho^{\prime}\right)+\bar{\gamma}_{F P}\left(\rho^{\prime}\right)\right) \bar{a}\left(\rho^{\prime}\right) \\
& \times \exp \left[-2 \int_{\rho}^{\rho^{\prime}} d \rho^{\prime \prime} \bar{\gamma}_{F P}\left(\rho^{\prime \prime}\right)\right]
\end{aligned}
$$

After obtaining this formal solution we then let the cut-off $\Lambda$ tend to $\infty$. As a consequence the boundary condition (4.28) is not necessarily satisfied since we are changing the order of limiting procedures.

Assume that the superconvergence relation (3.58) is satisfied so that $\bar{a}(\rho)$ vanishes identically, then we conclude from Eq. (4.31) that $C=\bar{C}(0)$ vanishes also. In other words, the condition (3.49) or $C=0$ follows from (3.58). Therefore, the superconvergence relation $Z_{3}^{-1}=0$ is the most fundamental condition for c.c., and it will be referred to as the CCC. This condition was first recognized as the condition for gluon confinement in 
the Landau gauge [27] since $Z_{3}$ had been known only in this gauge, but later this result was extended to other gauges and also to other colored particles [28].

It should be mentioned that although we have started from (4.28) the difference $\bar{C}(\rho)-$ $\bar{a}(\rho)$ does not necessarily vanish since $\bar{C}(\rho)$ and $\bar{a}(\rho)$ satisfy different RG equations. In particular, $C-Z_{3}^{-1}=\bar{C}(0)-\bar{a}(0)$ represents the contributions of the so-called GotoImamura-Schwinger term $[29,30]$. When $Z_{3}^{-1}=0$, however, this term should also vanish as we have remarked above [31]. We also have

$$
\begin{aligned}
& \delta\left(x_{0}-y_{0}\right)\left\langle 0\left|\left[\dot{A}_{\mu}^{a}(x), A_{\nu}^{b}(y)\right]\right| 0\right\rangle \\
= & -i \delta_{a b} \delta_{\mu \nu} Z_{3}^{-1} \delta^{4}(x-y)=0,
\end{aligned}
$$

and hence the vanishing of the Goto-Imamura-Schwinger term seems plausible as has been proved otherwise.

We conclude that c.c. is realized when $Z_{3}^{-1}=0$, although originally only gluons were considered to be confined. This CCC implies $C=0$, but we already know that $C \neq 0$ for abelian and broken non-abelian gauge symmetries. Therefore, in these cases $Z_{3}^{-1}$ cannot vanish either.

\section{Realization of Color Confinement}

In the preceding section we have obtained a simple but generic condition for c.c., then a natural question is raised of how to evaluate the renormalization constant $Z_{3}$ so that we know precisely in which theories c.c. is realized. It so happens that $Z_{3}$ can be evaluated exactly in QCD and we can readily check the CCC [28,32], but before entering this subject we have to discuss a more fundamental subject.

\subsection{Gauge-independence of the concept of color confinement}

In QED the renormalization constant $Z_{3}$ does not depend on the gauge parameter and is hence gauge-independent. It is not the case in QCD, however, and it is a function of the gauge parameter $\alpha$ and the gauge coupling constant $g$. Then, what is the significance of the CCC since $Z_{3}^{-1}$ might vanish in certain gauges but not in others?

In order to examine this question we first stress that the concept of color confinement is gauge-independent. When the condition (3.17) is satisfied, the only observable particles are hadrons, namely, color singlet bound states of quarks and gluons and they are represented by color singlet, and hence BRS invariant, composite operators when the LSZ reduction formula is applied. Then the only observable $\mathrm{S}$ matrix elements are the transition amplitudes among hadronic states.

Bearing this in mind we introduce the concept of the equivalence class of gauges [28,32]. Let us consider a class of Lagrangian densities $\left\{\mathcal{L}_{\alpha}\right\}$ representing a gauge theory such as 
QCD. Assume that all the members of this set are BRS invariant,

$$
\delta \mathcal{L}_{\alpha}=0,
$$

and further that the difference between any two members are exact, namely,

$$
\Delta \mathcal{L}=\mathcal{L}_{I I}-\mathcal{L}_{I}=\delta \mathcal{M},
$$

then this set $\left\{\mathcal{L}_{\alpha}\right\}$ is called an equivalence class of gauges. Lagrangian densities corresponding to different choices of $\alpha$ in (3.1) belong to the same equivalence class.

We introduce Green's functions in two gauges of the same class, then they are related to one another through the Gell-Mann-Low relation [33]:

$$
\left\langle A\left(x_{1}\right) B\left(x_{2}\right) \cdots\right\rangle_{I I}=\left\langle A\left(x_{1}\right) B\left(x_{2}\right) \cdots \exp (i \Delta S)\right\rangle_{I},
$$

where $A\left(x_{1}\right), B\left(x_{2}\right), \cdots$ are local operators, and

$$
\Delta S=\int d^{4} x \Delta \mathcal{L}=\int d^{4} x \delta \mathcal{M}
$$

In Eq. (5.3) we assume the convergence of the series expansion in powers of $\Delta S$.

In particular, when all the local operators are BRS invariant,

$$
\delta A=\delta B=\cdots=0,
$$

we have, as a result of Eq. (3.43), the equality

$$
\left\langle A\left(x_{1}\right) B\left(x_{2}\right) \cdots\right\rangle_{I I}=\left\langle A\left(x_{1}\right) B\left(x_{2}\right) \cdots\right\rangle_{I}
$$

subject to the convergence of the power series mentioned above. Thus Green's functions involving only BRS invariant operators do not depend on the choice of the gauge within the same equivalence class. When these composite operators represent hadrons we can apply the LSZ reduction formula [20] to such Green's functions to obtain the gauge-independent transition amplitudes for hadronic reactions [34,35,36].

The unitarity condition for the BRS invariant $\mathrm{S}$ matrix between two hadronic states $|a\rangle$ and $|b\rangle$ is given by

$$
\langle b \mid a\rangle=\left\langle b\left|S^{\dagger} S\right| a\right\rangle=\sum_{n}\left\langle b\left|S^{\dagger}\right| n\right\rangle\langle n|S| a\rangle,
$$

and a similar one for $S S^{\dagger}$. Color confinement is realized when the sum over intermediate states is saturated by hadronic states alone. Since the hadronic S matrix elements are gauge-independent, c.c. in one gauge automatically prevails in other gauges of the same equivalence class. This saturation of intermediate states by hadronic states could be employed as an alternative interpretation of c.c. since this statement is made in terms of observable hadrons alone. We shall come back to this new interpretation later in connection with the Meissner-like effect in the present approach. 


\subsection{Evaluation of $Z_{3}^{-1}$}

Next we shall proceed to evaluation of $Z_{3}^{-1}$ and for this purpose we shall study the RG equations for the running parameters $\bar{g}(\rho), \bar{\alpha}(\rho)$ and $\bar{\mu}(\rho)$,

$$
\begin{gathered}
\frac{d}{d \rho} \bar{g}(\rho)=\bar{\beta}(\rho), \\
\frac{d}{d \rho} \bar{\alpha}(\rho)=-2 \bar{\alpha}(\rho) \bar{\gamma}_{V}(\rho), \\
\frac{d}{d \rho} \bar{\mu}(\rho)=\bar{\mu}(\rho) .
\end{gathered}
$$

First, we shall define their asymptotic values by

$$
\bar{g}(\infty)=g_{\infty}, \bar{\alpha}(\infty)=\alpha_{\infty}, \bar{\mu}(\infty)=\infty .
$$

Asymptotic freedom (AF) is characterized by

$$
g_{\infty}=0
$$

and it is realized for $N_{f} \leq 16$.

By integrating (5.8b) we find

$$
\ln \frac{\alpha_{\infty}}{\alpha}=-2 \int_{0}^{\infty} d \rho \bar{\gamma}_{V}(\rho)
$$

or

$$
Z_{3}^{-1}=\exp \left[2 \int_{0}^{\infty} d \rho \bar{\gamma}_{V}(\rho)\right]=\frac{\alpha}{\alpha_{\infty}} .
$$

Thus evaluation of $Z_{3}^{-1}$ reduces to that of $\alpha_{\infty}$. If we identify $\alpha_{\infty}$ with the unrenormalized gauge parameter this is a rather trivial relation, but it is not trivial that $\alpha_{\infty}$ assumes only three possible values

$$
\alpha_{\infty}=-\infty, 0, \alpha_{0},
$$

where $\alpha_{0}$ is a numerical constant which depends only on the number of quark flavors as we shall see in the next subsection.

\subsection{Evaluation of $\alpha_{\infty}$}

Evaluation of $\alpha_{\infty}$ has been published elsewhere $[17,28,32]$ so that we shall be brief in what follows.

First, we introduce the $\beta$ function and the anomalous dimension $\gamma_{V}$ as series expansions in powers of the coupling constant: 


$$
\begin{gathered}
\beta(g)=g^{3}\left(\beta_{0}+\beta_{1} g^{2}+\cdots\right), \\
\gamma_{V}(g, \alpha)=g^{2}\left(\gamma_{0}(\alpha)+\gamma_{1}(\alpha) g^{2}+\cdots\right),
\end{gathered}
$$

where

$$
\begin{aligned}
\gamma_{0}(\alpha)= & \gamma_{00}+\gamma_{01} \alpha, \\
\gamma_{1}(\alpha)= & \gamma_{10}+\gamma_{11} \alpha+\gamma_{12} \alpha^{2}, \\
& \vdots
\end{aligned}
$$

The lowest order coefficients are given by

$$
\begin{aligned}
& \beta_{0}=-\frac{1}{32 \pi^{2}}\left(22-\frac{4}{3} N_{f}\right), \\
& \gamma_{00}=-\frac{1}{32 \pi^{2}}\left(13-\frac{4}{3} N_{f}\right), \gamma_{01}=\frac{3}{32 \pi^{2}}>0 .
\end{aligned}
$$

When $\beta_{0}$ is negative, namely, when $N_{f} \leq 16$, AF is realized, and we assume it in what follows. Then for large values of $\rho$ we obtain

$$
\bar{g}^{2}(\rho) \approx \frac{1}{b \rho} \cdot\left(b=-2 \beta_{0}>0\right)
$$

In order to check the convergence of the integral (5.11) we study the behavior of its integrand for large values of $\rho$ by expanding it in powers of $\bar{g}^{2}$, and we can easily verify the convergence when and only when

$$
\alpha_{\infty}=\alpha_{0}
$$

where $\alpha_{0}$ is defined by

$$
\gamma_{0}\left(\alpha_{0}\right)=\gamma_{00}+\gamma_{01} \alpha_{0}=0 .
$$

When the integral is divergent we obtain

$$
\alpha_{\infty}=-\infty, 0 .
$$

Apparently, the CCC (3.58) is satisfied when $\alpha_{\infty}=-\infty$ because of the sum rule (5.12), and we have to check if this case is actually realized in QCD. In order to check which of the three possible values of $\alpha_{\infty}$ in (5.13) is realized we have to study Eq. (5.8b) closely with the help of AF. In what follows we shall quote only the results.

Case 1) $\gamma_{00}<0, \alpha_{0}>0\left(N_{f}<10\right)$

$$
\alpha_{\infty}=\left\{\begin{array}{c}
\alpha_{0}, \quad \text { for } \alpha>0 \\
0, \quad \text { for } \alpha=0 \\
-\infty, \quad \text { for } \alpha<0
\end{array}\right.
$$


Case 2) $\gamma_{00}>0, \alpha_{0}<0\left(10 \leq N_{f} \leq 16\right)$

$$
\alpha_{\infty}=\left\{\begin{array}{c}
0, \quad \text { for } \alpha>\alpha_{0}+h\left(g^{2}\right), \\
\alpha_{0}, \quad \text { for } \alpha=\alpha_{0}+h\left(g^{2}\right), \\
-\infty, \quad \text { for } \alpha<\alpha_{0}+h\left(g^{2}\right),
\end{array}\right.
$$

where

$$
\alpha=\alpha_{0}+h\left(g^{2}\right)=\alpha_{0}+g^{2}\left(h_{0}+h_{1} g^{2}+\cdots\right)
$$

is a special solution of the following equation:

$$
\frac{d \alpha}{d g}=-2 \alpha \frac{\gamma_{V}(g, \alpha)}{\beta(g)} .
$$

Thus in both cases $\alpha_{\infty}=-\infty$ is realized when the gauge parameter and the gauge coupling constant are properly chosen and consequently the CCC is satisfied.

A domain in the $\left(\alpha, g^{2}\right)$ half-plane corresponding to $\alpha_{\infty}$ is denoted by $D\left(\alpha_{\infty}\right)$, then this half-plane is covered by $\bar{D}(-\infty), \bar{D}(0), \bar{D}\left(\alpha_{0}\right)$, where the bar denotes closure. The above arguments show that c.c. is realized in $D(-\infty)$, but what happens in the other two domains? Formally we can apply the argument on the gauge-independence of the concept of c.c., namely, for two different values of $\alpha$ we have

$$
\Delta \mathcal{L}=\frac{1}{2}(\Delta \alpha) B \cdot B=-\frac{i}{2}(\Delta \alpha) \delta(\bar{c} \cdot B)
$$

or

$$
\mathcal{M}=-\frac{i}{2}(\Delta \alpha)(\bar{c} \cdot B)
$$

so that we can utilize the formula (5.6) to show that c.c. prevails in the other two domains. In this case $Z_{3}^{-1}=0$ is a sufficient condition but not a necessary one for c.c. since it is not satisfied in the two domains $D(0)$ and $D\left(\alpha_{0}\right)$. The identity (5.3) is valid only when the series expansion in powers of $\Delta S$ is convergent. In the present case we have to check the convergence of the power series in $\Delta \alpha$. Then Eq. (4.9) shows within the framework of the RG approach that this implies the convergence of the power series in $\Delta \bar{\alpha}(\rho)$. Let us consider, for instance, the case 1$)$, then the line $\alpha=0$ is the border between $D(-\infty)$ and $D\left(\alpha_{0}\right)$. Let us introduce $\alpha_{1}<0$ and $\alpha_{2}>0$, then even when $|\Delta \alpha|=\left|\alpha_{2}-\alpha_{1}\right| \ll 1$, $\Delta \bar{\alpha}(\rho)$ tends to $\infty$ in the limit $\rho \rightarrow \infty$ and the convergence of the power series turns out to be doubtful thereby suggesting that $\alpha=0$ would be a branch point of Green's functions involving BRS variant operators. This, in turn, means that such Green's functions would be multi-valued functions of $\alpha$, Then a question is raised of whether Green's functions involving only BRS invariant operators be also multi-valued. When this were the case c.c. might be realized only in the domain $D(-\infty)$, so that $Z_{3}^{-1}=0$ would represent a 
necessary and sufficient condition for c.c., but it has not been clarified yet if it would really be the case.

We may close this section by concluding that c.c. is realized in QCD provided that color symmetry is not spontaneously broken and AF is valid.

\section{Consequences of the $\mathrm{CCC}$}

In this section we shall show how the $\mathrm{CCC}, Z_{3}^{-1}=0$, is related to other interpretations of confinement based mainly on the dual Meissner effect. Since the starting point of other interpretations are quite distinct from that of the present approach it is not easy to compare the basic formulations for the purpose of clarifying their relationships. Therefore, we shall try to compare the consequences of our approach with those of the others.

An attempt has been made, however, by one of the present authors (M.C.) and Kobayashi [37] to clarify the relationship between the Seiberg formulation $[38,39,40]$ and the present superconvergence rule in the Landau gauge by studying the criteria for confinement expressed in terms of the $\beta$ function and the anomalous dimension of the gauge field.

\subsection{An intuitive interpretation of the CCC}

Let us consider a dielectric medium and put a positive test charge inside, then negative charges are attracted and positive ones are repelled by it. Therefore, it induces a new charge distribution in the medium and the total charge inside a sphere of radius $r$ around the test charge is a function of $r$. It is denoted by $\bar{e}(r)$ and called the running charge.

In classical physics the vacuum means the empty mathematical space or the void, but in quantum physics the physical vacuum is a kind of medium with a rich structure and has to be distinguished from the classical vacuum or the mathematical space. The dielectric constant is defined relative to one of the vacua. Now let us regard the physical vacuum as a dielectric medium and call the test charge $e_{0}=\bar{e}(0)$ the bare charge and the total charge inside a sphere of a sufficiently large radius $e=\bar{e}(\infty)$ the renormalized charge.

We then introduce the dielectric constant of the physical vacuum $\epsilon$ relative to the mathematical one and write down the static Coulomb potential between two electrons in two alternative ways,

$$
V(r)=\frac{e^{2}}{4 \pi r}=\frac{e_{0}^{2}}{4 \pi \epsilon r}
$$

We also introduce the renormalization constant $Z_{3}$ of the electromagnetic field, then we find

$$
e_{0}^{2}=Z_{3}^{-1} e^{2}
$$


Thus we are led to

$$
\epsilon=Z_{3}^{-1} \text {. }
$$

At the end of Section 2 we have discussed the two-phase structure or the emergence of the Meissner effect in the limit $\epsilon \rightarrow 0$, and now through Eq. (6.3) we find that this condition is equivalent to the CCC. Furthermore, we find that this condition along with (6.2) implies $e_{0}=0$ or AF. In the extreme case of $\epsilon=0$, a small test charge would attract an unlimited amount of like charges around it leading the system into a catastrophic state of infinite charge. Nature would take safety measures to prevent such a state from emerging, and a possible resolution to avoid it would be to bring another test particle of opposite charge. The total charge of the whole system is then equal to zero and charge confinement would be realized.

The dielectric constant of the vacuum $\epsilon$ is larger than unity, however, as a consequence of the screening effect due to vacuum polarization. Quantum mechanically $Z_{3}^{-1}$ is larger than unity because of the contributions of charged particles of positive-definite metric so that the condition $Z_{3}^{-1}=0$ could be realized only in the presence of charged particles of indefinite metric. They do not appear in QED unless they are put by hand just as the magnetic monopoles in Section 2, however, so that electric charges cannot be confined in QED. Indeed, this is a conclusion repeatedly drawn, but QED is a good laboratory for Gadankenexperiment, however fictitious, to illustrate the mechanism of confinement.

\subsection{The CCC and the linear potential}

In this subsection we shall give an intuitive argument on a possible connection between the CCC and Wilson's area law in the lattice gauge theory [6].

Wilson has formulated the criterion for confinement in terms of a loop correlation function defined by

$$
W[C]=\operatorname{Tr} P \exp \left(i g \int_{C} A_{\mu} d x_{\mu}\right),
$$

where $P$ stands for the path ordering. $W$ creates a tube of flux, along the path $C$, of strength equal to one quark color charge. a quark-antiquark pair is attached to both ends of the open path or the path is closed. The average value of this expression is defined in terms of path integrals in Euclidean space, and for a large closed path $C$, its average value is asymptotically proportional to

$$
\langle W\rangle \propto \exp (- \text { perimeter })
$$

or

$$
\langle W\rangle \propto \exp (- \text { area })
$$


The perimeter and the area denote those of the path $C$, respectively. Wilson's criterion for confinement is the realization of the latter case called the area law.

In evaluating the loop correlation function (6.4) we shall choose a rectangular contour with a temporal extension $T$ and a spatial extension $R$, and the effective potential between a heavy quark and a heavy antiquark is given by

$$
V[R]=-\lim _{T \rightarrow \infty} \frac{1}{T} \ln W[R \times T] .
$$

When the area law (6.6) is obeyed we have the following linear potential at large distances:

$$
V[R]=\sigma R .
$$

The linear potential is no longer valid, however, when a quark-antiquark pair can be created from the vacuum, since it is energetically more favorable to split the tube of flux between the heavy quark pair thereby attaching a light quark and a light antiquark pair to the two split ends than to stretch the string indefinitely. We shall realize this point later in this subsection.

In evaluating the expression (6.7) we shall introduce a cluster expansion as given by

$$
\begin{aligned}
& \ln \left\langle\operatorname{Tr} P \exp \left(i g \oint_{C} A_{\mu} d x_{\mu}\right)\right\rangle \\
= & -\frac{1}{2} g^{2} \oint_{C} \oint_{C}\left\langle A_{\mu}(x) A_{\nu}(y)\right\rangle d x_{\mu} d y_{\nu}+\cdots .
\end{aligned}
$$

Since we are taking the limit $T \rightarrow \infty$ for the path $C=R \times T$ we may keep only the temporal path, and we obtain

$$
V[R] \propto \int d x_{0} D_{F}(x)_{00},
$$

where $D_{F}(x)_{\mu \nu}$ is the gluon propagator in Eq. (3.54), and we may insert the representations (3.55) and (3.56) into the integrand.

When the CCC is satisfied we may express $D\left(k^{2}\right)$ as

$$
D\left(k^{2}\right)=\int d m^{2} \frac{\tau\left(m^{2}\right)}{\left(k^{2}+m^{2}-i \epsilon\right)^{2}},
$$

where

$$
\tau\left(m^{2}\right)=\int_{0}^{m^{2}} d M^{2} \rho\left(M^{2}\right)
$$

with

$$
\tau(0)=\tau(\infty)=0 .
$$

Now we switch to a rather crude approximation since the concept of the static potential itself is phenomenological in nature. Actually $r=0$ is a singularity in the potential. 
Although the CCC suppresses the $1 / r$ singularity we are still left with a mild singularity at $r=0$, and this forces us to introduce a crude approximation. Let us assume that $\left|\tau\left(m^{2}\right)\right|$ has a maximum at $m^{2}=\kappa^{2}$, then because of asymptotic freedom gauge interactions are stronger at lower energies than at higher energies and $\kappa$ represents a typical mass of unconfined systems sharing the same set of quantum numbers with a single gluon. Thus we may further assume that $\kappa$ is of the order of or even smaller than the pion mass. Then we may approximate (6.11) by a smeared dipole type propagator

$$
D\left(k^{2}\right) \approx \frac{f}{\left(k^{2}+\kappa^{2}-i \epsilon\right)^{2}} .
$$

Then the static potential $V_{q \bar{q}}(r)$ between a heavy quark and a heavy antiquark can be evaluated by utilizing (6.10) as

$$
\begin{aligned}
V_{q \bar{q}}(r) & \propto \frac{1}{\kappa} e^{-\kappa r} \\
& \cong \frac{1}{\kappa}-r, \text { for } r \ll 1 / \kappa .
\end{aligned}
$$

Thus we have a linear potential in a limited range. The first constant term may be included in the self energies of the heavy quarks $[41,42]$. An unlimited extension of a linear potential to large distances would lead to too strong a van der Waals force between hadrons [43] so that the potential should cease to be linear and fall off at large distances as remarked in the beginning of this subsection. In this connection it should be mentioned that the approximate linear potential is a result of but not the cause of color confinement as we have seen already.

The $q \bar{q}$ interactions discussed here are generated by exchanging colored objects and has a relatively long range. This indicates, however, that vortices connecting a quarkantiquark pair should have a finite length of the order of or less than $1 / \kappa$. As we shall see in the next subsection the hadron interactions are generated by exchanging color singlet hadrons and are of the short-range type.

\subsection{Hadron interactions and the Meissner-like effect}

In the Subsection 6.1 we have discussed the emergence of the two-phase structure and the related dual Meissner effect. This is expected to take place in the limit of the vanishing dielectric constant. In real QED, however, it is larger than unity and this scenario fails to be realized. For instance, let us consider the interaction between two electrically neutral systems. Then, their interaction is given by the van der Waals potential

$$
V_{v d W}(r) \propto r^{-6}
$$

This shows that the electric field generated by neutral systems penetrate into the vacuum without any sharp cut-off indicating the failure of the dual Meissner effect. 
In QCD we can elucidate the dynamics of hadrons by making reference to dispersion relations. It has been clarified by Oehme [44] that dispersion relations for the scattering of hadrons remain applicable provided that confinement excludes quarks and gluons from the physical state vector space. This is precisely one of the consequences of the CCC, and we can extract necessary information about hadron interactions from dispersion relations on the assumption that the complete hadron spectrum can be accounted for by QCD.

Let us consider the nucleon-nucleon scattering as an example, then the potential between them is given by the pole contributions in the crossed channels. The least massive hadron that can be exchanged between them is the pion, and the resulting interaction is represented by the Yukawa potential,

$$
V_{Y}(r) \propto \frac{e^{-\mu r}}{r}
$$

where $\mu$ denotes the pion mass.

When we compare this result with the van der Waals potential we recognize that the flux of the color gauge field emerging from color singlet nucleons cannot penetrate into the confining vacuum leaving no trace of long range forces and that the penetration depth is given by the pion Compton wave length. Thus we notice that the Yukawa mechanism of generating the nuclear forces bears a strong resemblance to the Meissner effect in the type II superconductor.

Although we have chosen a rather abstract approach to c.c. on the basis of BRS symmetry and asymptotic freedom, it shares essentially the same salient features with other approaches in that the vacuum allows penetration of the chromoelectric flux from hadrons only by a finite depth into it.

To conclude we stress that all these characteristic features of confinement are the consequences of the CCC, namely, $Z_{3}^{-1}=0$.

\section{Acknowledgements}

This work is partially supported by the Academy of Finland under the Project No. 163394. We are grateful to W.-F. Chen and C. Montonen for useful comments.

\section{Appendix A: Derivation of Dighost Bound States from the Condition (3.49)}

In this appendix we shall prove the existence of the dighost bound states on the basis of the condition (3.49) by utilizing the properties of the representation of the BRS charge $[16,17]$.

Let us introduce a complete set of basis $\left\{\left|e_{i}\right\rangle\right\}$ in the state vector space $\mathcal{V}$ and define the matrix $\eta$ by 


$$
\eta_{i j}=\left\langle e_{i} \mid e_{j}\right\rangle
$$

Then, due to the properties of the inner product, it is hermitian,

$$
\eta^{\dagger}=\eta
$$

Provided that $\mathcal{V}$ is non-degenerate we can always choose the set of basis so as to satisfy the constraint

$$
\eta^{2}=1
$$

which defines the standard form of $\eta$.

The representation $t$ of a given linear operator $T$ with respect to the given complete set of basis is defined by

$$
T\left|e_{i}\right\rangle=\sum_{j}\left|e_{j}\right\rangle t_{j i}
$$

and the hermitian conjugate $T^{\dagger}$ is defined by

$$
\langle k|T| l\rangle=\langle l|T| k\rangle^{*},
$$

for an arbitrary pair of states $|l\rangle$ and $|k\rangle$. Then the representation of $T^{\dagger}$ denoted by $\tilde{t}$ satisfies the relation

$$
(\eta t)^{\dagger}=\eta \tilde{t}
$$

The BRS charge $Q_{B}$ is hermitian and nilpotent as discussed in Section 3, and its representation $q$ satisfies

$$
q^{2}=0, \quad q^{\dagger}=\eta q \eta
$$

Now we can introduce the Hodge decomposition of $\mathcal{V}$ as

$$
\mathcal{V}=\mathcal{V}_{s}+\mathcal{V}_{d}+\mathcal{V}_{p}
$$

The typical members of these subspaces satisfy the following relations:

$$
\begin{aligned}
& q|s\rangle=0, q^{\dagger}|s\rangle=0, \quad \text { for }|s\rangle \in \mathcal{V}_{s} \text { (harmonic), } \\
& q|d\rangle=0, q^{\dagger}|d\rangle \neq 0, \text { for }|d\rangle \in \mathcal{V}_{d} \text { (exact), } \\
& q|p\rangle \neq 0, q^{\dagger}|p\rangle=0, \quad \text { for }|p\rangle \in \mathcal{V}_{p} \text { (co-exact). }
\end{aligned}
$$

In each subspace we introduce a complete set of basis such as $\left\{\left|s_{i}\right\rangle\right\},\left\{\left|d_{i}\right\rangle\right\}$ and $\left\{\left|p_{i}\right\rangle\right\}$ satisfying 


$$
\begin{aligned}
& \left\langle s_{i} \mid s_{j}\right\rangle=\left\langle d_{i} \mid p_{j}\right\rangle=\delta_{i j}, \\
& \left\langle d_{i} \mid d_{j}\right\rangle=\left\langle p_{i} \mid p_{j}\right\rangle=0 .
\end{aligned}
$$

With these preliminaries we shall start from an analogue of (3.34),

$$
\left\langle\chi^{a}(x), \bar{\beta}^{b}(y)\right\rangle=\delta_{a b} D_{F}(x-y),
$$

where $\bar{\beta}$ is the asymptotic field of $\bar{B}$. Then, by making use of (3.43) and (3.11b) we find

$$
\left\langle\bar{\delta} \chi^{a}(x), c^{b}(y)^{i n}\right\rangle=i \delta_{a b} D_{F}(x-y) .
$$

When the condition (3.49) is valid we have

$$
\bar{\delta} \chi^{a}(x)|0\rangle \in \mathcal{V}_{s}+\mathcal{V}_{d}
$$

However, $\bar{\delta} \chi^{a}(x)|0\rangle$ has zero norm as seen from

$$
\left\langle 0\left|\bar{\delta} \chi^{a}(x) \bar{\delta} \chi^{a}(x)\right| 0\right\rangle=\left\langle 0\left|\bar{\delta}\left(\chi^{a}(x) \bar{\delta} \chi^{a}(x)\right)\right| 0\right\rangle=0,
$$

where we have used (3.43) for $\bar{Q}_{B}$. Hence we conclude

$$
\bar{\delta} \chi^{a}(x)|0\rangle \in \mathcal{V}_{d}
$$

The orthogonality conditions (A.10) and (A.12) then imply that $c^{b}(y)^{i n}|0\rangle$ should involve a non-vanishing co-exact component and hence

$$
\delta c^{b}(y)^{i n}|0\rangle=-\frac{1}{2} g(c(y) \times c(y))^{b, i n}|0\rangle \neq 0 .
$$

This establishes the existence of the dighost bound states.

\section{Appendix B: Proof of the Condition (3.45) for Colored Par- ticles}

When the condition (3.49) is satisfied we can prove (3.44) and hence (3.45) for colored particles. For this purpose we have to derive (3.48) by applying the reduction formula to the identity (3.41). In the absence of the asymptotic fields, however, we have to devise a new proof.

We start from the Lehmann representation [23] for a complex scalar field,

$$
\begin{aligned}
\Delta_{F}^{\prime}(x-y) & =\left\langle 0\left|T\left[\phi(x) \phi^{\dagger}(y)\right]\right| 0\right\rangle \\
& =\int_{m^{2}}^{\infty} d M^{2} \rho\left(M^{2}\right) \Delta_{F}\left(x-y, M^{2}\right),
\end{aligned}
$$


where $m$ denotes the mass of the quantum of this field, and

$$
\Delta_{F}\left(x, M^{2}\right)=\frac{-i}{(2 \pi)^{4}} \int d^{4} p e^{i p \cdot x} \Delta_{F}\left(p, M^{2}\right)
$$

where

$$
\Delta_{F}\left(p, M^{2}\right)=\left(p^{2}+M^{2}-i \epsilon\right)^{-1} .
$$

There are two alternative possibilities for the nature of the spectral function $\rho\left(M^{2}\right)$ :

case i)

$$
\rho\left(M^{2}\right)=\delta\left(M^{2}-m^{2}\right)+\sigma\left(M^{2}\right) \theta\left[M^{2}-(m+\mu)^{2}\right], \mu \neq 0 .
$$

In this case there is a pole term in the propagator on the mass shell.

case ii)

There is no pole term due to infrared singularities and $\mu=0$ in this case.

In the case i) there are asymptotic fields defined by

$$
\begin{aligned}
\phi(x) & =\phi^{i n}(x)-\int d^{4} y \Delta_{R}(x-y) \cdot K_{y} \phi(y) \\
& =\phi^{\text {out }}(x)-\int d^{4} y \Delta_{A}(x-y) \cdot K_{y} \phi(y),
\end{aligned}
$$

where $K_{y}=\square_{y}-m^{2}$ denotes the Klein-Gordon operator, and the retarded and advanced functions $\Delta_{R}$ and $\Delta_{A}$, respectively, satisfy the following equations:

$$
K_{x} \Delta_{R}(x)=K_{x} \Delta_{A}(x)=-\delta^{4}(x) .
$$

In the case ii) the mass shell is a branch-cut in the propagator and there are no discrete or normalizable single particle states. Instead of a single particle state, for instance, we have a superposition of multi-photon states like a wave packet. Also, as clarified by Bloch and Nordsieck [45], electron scattering is an inclusive reaction since an electron is accompanied by an infinite number of infrared photons which escape detection. In this way we have only a limited class of observable quantities in the infared singular theories.

Now we turn to the reduction formula in the case i). The general idea is to replace a propagator by a single particle wave function. For instance,

$$
\left\langle 0\left|T\left[\phi(x) \phi^{\dagger}(y)\right]\right| 0\right\rangle \rightarrow\left\langle s\left|\phi^{\dagger}(y)\right| 0\right\rangle .
$$

We shall call this operation $\mathcal{R}_{s}$ and it is realized by 


$$
\begin{aligned}
\mathcal{R}_{s}\left\langle 0\left|T\left[\phi(x) \phi^{\dagger}(y)\right]\right| 0\right\rangle & =\int d^{4} x\left\langle s\left|\phi^{\dagger}(x)\right| 0\right\rangle(-i) K_{x}\left\langle 0\left|T\left[\phi(x) \phi^{\dagger}(y)\right]\right| 0\right\rangle \\
& =\left\langle s\left|\phi^{\dagger}(y)\right| 0\right\rangle,
\end{aligned}
$$

or generally,

$$
\mathcal{R}_{s}\langle 0|T[\phi(x) A B \cdots]| 0\rangle=\langle s|T[A B \cdots]| 0\rangle .
$$

When $T$ is replaced by the antichronological symbol $\tilde{T},(-i) K_{x}$ should be replaced by $i K_{x}$.

Amplitudes are not what we can directly observe; so we shall study their absolute squares and expectation values. The reduction formula for the former should realize the following replacement:

$$
\begin{aligned}
& \left\langle 0\left|\tilde{T}\left[\phi(u) \phi^{\dagger}(v)\right]\right| 0\right\rangle\left\langle 0\left|T\left[\phi(x) \phi^{\dagger}(y)\right]\right| 0\right\rangle \\
& \rightarrow \sum_{s}\langle 0|\phi(u)| s\rangle\left\langle s\left|\phi^{\dagger}(y)\right| 0\right\rangle \\
& \rightarrow \int_{m^{2}}^{(m+\lambda)^{2}} d M^{2} \rho\left(M^{2}\right) i \Delta^{+}\left(u-y, M^{2}\right)
\end{aligned}
$$

where $\lambda$ depends on the experimental condition and corresponds to the Bloch-Nordsieck states in the case 2). In the case 1) the reduction formula is given, for $\lambda<\mu$, by

$$
\begin{aligned}
& \sum_{s} \int d^{4} x d^{4} y\left\langle 0\left|\tilde{T}\left[\ldots \phi^{\dagger}(y)\right]\right| 0\right\rangle i \overleftarrow{K}_{y}\langle 0|\phi(y)| s\rangle \\
& \times\left\langle s\left|\phi^{\dagger}(x)\right| 0\right\rangle(-i) K_{x}\langle 0|T[\phi(x) \ldots]| 0\rangle \\
& =\sum_{s}\langle 0|\tilde{T}[\ldots]| s\rangle\langle s|T[\ldots]| 0\rangle .
\end{aligned}
$$

In this case the result does not depend on $\lambda$ and we may take the limit $\lambda \rightarrow 0$.

By the same token the reduction formula for expectation values is given, typically, by

$$
\begin{aligned}
& \int d^{4} x d^{4} y\left\langle s\left|\phi^{\dagger}(x)\right| 0\right\rangle(-i) \vec{K}_{y}\left\langle 0\left|T\left[\phi(y) J(z) \phi^{\dagger}(y)\right]\right| 0\right\rangle \times(-i) \overleftarrow{K}_{x}\langle 0|\phi(x)| s\rangle \\
& =\langle s|J(z)| s\rangle
\end{aligned}
$$

In order to proceed to infrared singular theories we shall discuss QED in what follows.

We introduce the electron propagator $S_{F}(p)$ and put

$$
(i \gamma \cdot p+m) S_{F}(p)=a\left(p^{2}\right)+\frac{i \gamma \cdot p}{m} b\left(p^{2}\right) .
$$


Then for the free electron field we have $a=1$ and $b=0$. The behavior of the propagator near the mass shell is given by

$$
a\left(p^{2}\right), b\left(p^{2}\right) \propto\left(1+\frac{p^{2}}{m^{2}}\right)^{c}, \text { with } c=-(3-\alpha) \frac{e^{2}}{8 \pi^{2}} .
$$

This result was obtained by solving Ovsianikov's equation in RG [46]. It shows that the mass shell $p^{2}+m^{2}=0$ is a branch-cut. Now define

$$
D(\partial)=-i \gamma \cdot \partial-m, \quad \tilde{D}(\partial)=i \gamma \cdot \partial-m .
$$

In the absence of the branch-cut we have

$$
\begin{aligned}
& \int d^{4} x d^{4} y\langle s|\bar{\psi}(x)| 0\rangle(-i) D\left(\partial_{x}\right)\langle 0|T[\psi(x) J(z) \bar{\psi}(y)]| 0\rangle \\
& \times(-i) \tilde{D}\left(\overleftarrow{\partial}_{y}\right)\langle 0|\psi(y)| s\rangle \\
& =\langle s|J(z)| s\rangle
\end{aligned}
$$

In special cases we know the expectation values $\langle J\rangle$ a priori. For instance, the electric charge of the wave-packet-like state $|s\rangle$ is known to be equal to $-e$, and $J$ should be replaced by the space integral of the time component of $J_{\mu}$,

$$
Q=\int d^{3} z J_{0}(z)
$$

where $J_{\mu}$ represents the 4 -dimensional electric current. In this case $\langle Q\rangle$ is independent of the parameter $\lambda$ indicating the cancellation of the branch-cut between the propagator and the vertex function provided that they are related to one another through the Ward identity (3.47).

Since we have two electron propagators in the Feynman diagram corresponding to $\left\langle\psi J_{\mu} \bar{\psi}\right\rangle$, we have to eliminate one of them by multiplying $\mathcal{D}(\partial)$ instead of $D$, where

$$
\mathcal{D}(i p) S_{F}(p)=-1 .
$$

By taking the limit $\lambda \rightarrow 0$ we have

$$
\begin{aligned}
& \int d^{4} x d^{4} y\langle s|\bar{\psi}(x)| 0\rangle(-i) \mathcal{D}\left(\partial_{x}\right)\left\langle 0\left|T\left[\psi(x) J_{\mu}(z) \bar{\psi}(y)\right]\right| 0\right\rangle \\
& \times(-i) \tilde{D}\left(\overleftarrow{\partial}_{y}\right)\langle 0|\psi(y)| s\rangle \\
& =\left\langle s\left|J_{\mu}(z)\right| s\right\rangle C .
\end{aligned}
$$


Since the normalization of the wave-packet-like state does not guarantee the proper normalization of the "on-shell-limit state", we have introduced the constant $C$. It has to be determined by utilizing the known value of $\langle Q\rangle$. In QED we have

$$
C=1+(3-\alpha) \frac{e^{2}}{8 \pi^{2}} .
$$

In the absence of the asymptotic fields we can apply the same procedure to QCD and obtain the result (3.48) from the identity (3.14) provided that $M_{\mu}$ represents the spin zero projection of $\delta \bar{\delta} A_{\mu}[27,28]$ and $|\gamma\rangle$ the on-shell-limit of a single quark or a single gluon state in the sense of Bloch and Nordsieck.

\section{References}

[1] H. B. Nielsen and P. Olesen, Nucl. Phys. B6 , 45 (1973).

[2] Y. Nambu, Phys. Rev. D10 , 4262 (1974).

[3] S. Mandelstam, Phys. Reports C23 , 245 (1976).

[4] G. 't Hooft, High Energy Physics, Proceedings of the European Physical Society International Conference, edited by A. Zichichi, Editorice Compositori, Bologna, 1976, p. 1225 .

[5] G. 't Hooft, Nucl. Phys. B79 , 276 (1974).

[6] K. G. Wilson, Phys. Rev. D14, 2455 (1974).

[7] L. Susskind and K. Kogut, Phys. Rev. D11 , 395 (1975).

[8] N. Seiberg and E. Witten, Nucl. Phys. B426 , 19 (1994); ibid. B431 , 484 (1994).

[9] T. D. Lee, Particle Physics and Introduction to Field Theory, Harwood Academic Publ. Chur-London-New York, 1981, Chapter 17.

[10] C. Becchi, A. Rouet and R. Stora, Ann. Phys. 98 , 287 (1976).

[11] D. J. Gross and F. Wilczek, Phys. Rev. Lett. 30 , 1343 (1973).

[12] H. D. Politzer, Phys. Rev. Lett. 30 , 1346 (1973).

[13] L. D. Faddeev and V. N. Popov, Phys. Lett. 25B , 29 (1967).

[14] T. Kugo and I. Ojima, Prog. Theor. Phys. Suppl. No. 66 , 1 (1979).

[15] K. Nishijima, Nucl. Phys. B238 , 601 (1984). 
[16] K. Nishijima, Prog. Theor. Phys. 80 , 897 and 905 (1988).

[17] K. Nishijima, Czech. J. Phys. 46 , 1 (1996).

[18] R. Marnelius, Nucl. Phys. B372 , 218 (1992).

[19] K. Nishijima, Field Theory (in Japanese), Kinokuniya, Tokyo, 1987, as quoted in N. Nakanishi and I. Ojima, Covariant Operator Formalism of Gauge Theories and Quantum Gravity, World Scientific, Singapore, 1991.

[20] H. Lehmann, K. Symanzik and W. Zimmermann, Nuovo Cimento 1 , 205 (1955).

[21] K. Nishijima and Y. Okada, Prog. Theor. Phys. 72 , 294 (1984).

[22] K. Nishijima, Prog. Theor. Phys. 74 , 889 (1985).

[23] H. Lehmann, Nuovo Cimento 11 , 342 (1954).

[24] R. Oehme and W. Zimmermann, Phys. Rev. D21 , 471 and 1661 (1980).

[25] K. Nishijima, Prog. Theor. Phys. 75 , 1221 (1986).

[26] R. Oehme, Phys. Lett. 195B , 60 (1987).

[27] K. Nishijima, Int. J. Mod. Phys. A9 , 3799 (1994).

[28] K. Nishijima, Int. J. Mod. Phys. A10 , 3155 (1995).

[29] T. Goto and T. Imamura, Prog. Theor. Phys. 14, 396 (1955).

[30] J. Schwinger, Phys. Rev. Lett. 3 , 296 (1959).

[31] M. Chaichian and K. Nishijima, "The Goto-Imamura-Schwinger Term and Renormalization Group", Invited Talk at the Bogolyubov Conference, Moscow-Dubna-Kiev, 27 Sept. - 6. Oct. 1999.

[32] K. Nishijima and N. Takase, Int. J. Mod. Phys. A11, 2281 (1996).

[33] M. Gell-Mann and F. Low, Phys. Rev. 84 , 350 (1951).

[34] R. Haag, Phys. Rev. 112 , 669 (1958).

[35] K. Nishijima, Phys. Rev. 111 , 995 (1958).

[36] W. Zimmermann, Nuovo Cimento 10 , 597 (1958).

[37] M. Chaichian and T. Kobayashi, Phys. Lett. B481 , 26 (2000). 
[38] N. Seiberg, Phys. Rev. D49 , 6857 (1994).

[39] N. Seiberg, Nucl. Phys. B435 , 129 (1995).

[40] K. Intriligator and N. Seiberg, Nucl. Phys. Proc. Suppl. 45BC , 1 (1996).

[41] A.M. Polyakov, Nucl. Phys. B164 , 2445 (1979).

[42] V.S. Dotsenko and S.N. Vergeles, Nucl. Phys. B169 , 527 (1980).

[43] S. Matsuyama and H. Miyazawa, Prog. Theor. Phys. 61 , 942 (1979).

[44] R. Oehme, "Analytic Structure of Amplitudes in Gauge Theories with Confinement", Invited Talk at the Conference on New Problems in the General Theory of Fields and Particles of the International Congress of Mathematical Physics, La Sorbonne, Paris, July 1994.

[45] F. Bloch and A. Nordsieck, Phys. Rev. 52, 54 (1937).

[46] N. N. Bogoliubov and D. V. Shirkov, Introduction to the Theory of Quantized Fields, Interscience Publ., New York, 1959. Chapter 8. 